\title{
Antioxidant capacity of banana cultivar 'Nipah' (Musa acuminate balbisiana) extracted with different solvents
}

\begin{abstract}
Antioxidant is one of the biological effects from main phenolic components of fruit such as anthocyanins, flavonols, flavones, isoflavones, flavonones, and catechins. Antioxidant test is promoted in the research study of horticulture and food science to find fruit antioxidant capacity. Different solvents and percentages have different polarities that used in extraction will effects yield obtained and resulting antioxidant activities. Antioxidant capacity for banana specifically cultivar 'Nipah' (Musa acuminate balbisiana) were studied for its total phenolic content and DPPH radical (1,1-diphenyl-2-picrylhydrazyl). The three solvents that used were isacetone, methanol, ethanol in three different concentrations (50, 70 and 90\%). The antioxidant capacity helps to determine early or initial fresh-cut sample of 'Nipah' banana properties. Evaluated using phenolic contents andgallic acid as a standard solution $(10,25$, 50, 75 and $100 \mathrm{mg} \mathrm{L}-1, \mathrm{r} 2=\geq 0.900$ ) and 2-2-diphenyl-1-picrylhydrazyl (DPPH) radicalscavenging assay against control absorbance. Antioxidant capacities of 'Nipah' banana extracts tested varied. Acetone 50\% yield 122.9 GAE 100 g-1 (fw) has the highest polyphenol content and is an efficient solvent for extraction. The higher the correlation of the total phenolic content of the fruit, the higher the DPPH values too. High phenol content determines the high antioxidant capacity of the fruit. Hence, once the evaluation of antioxidant capacity for 'Nipah' banana using best solvent is identified, values are added to 'Nipah' banana to be one of the local antioxidant fruits.
\end{abstract}

Keyword: Antioxidant capacity; 'Nipah' banana; Total phenolic content; DPPH 